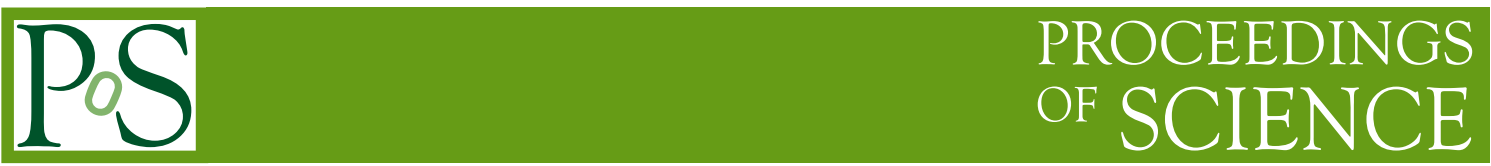

\title{
DarkSide-50: status of the detector and results
}

Yann Guardincerri*1 , P. Agnes ${ }^{2}$, I. F. M. Albuquerque ${ }^{41}$, T. Alexander ${ }^{48,1,30}$, A. K. Alton ${ }^{5}$, D. M. Asner ${ }^{30}$, H. O. Back ${ }^{30}$, B. Baldin ${ }^{1}$, K. Biery ${ }^{1}$, V. Bocci ${ }^{35}$, G. Bonfini ${ }^{4}$, W. Bonivento ${ }^{8}$, M. Bossa ${ }^{3,4}$, B. Bottino ${ }^{14,13}$, A. Brigatti ${ }^{25}$, J. Brodsky ${ }^{34}$, F. Budano ${ }^{39,40}$, S. Bussino ${ }^{39,40}$, M. Cadeddu ${ }^{9,8}$, L. Cadonati ${ }^{48}$, M. Cadoni ${ }^{9,8}$, F. Calaprice ${ }^{34}$, N. Canci ${ }^{16,4}$, A. Candela ${ }^{4}$, M. Caravati ${ }^{9,8}$, M. Cariello $^{13}$, M. Carlini ${ }^{4}$, S. Catalanotti ${ }^{29,28}$, P. Cavalcante ${ }^{4}$, A. Chepurnov ${ }^{27}$, C. Cicalò ${ }^{8}$, A. G. Cocco $^{28}$, G. Covone ${ }^{29,28}$, D. D'Angelo ${ }^{26,25}$, M. D'Incecco ${ }^{4}$, S. Davini ${ }^{3,4,13}$, S. De Cecco $^{22}$, M. De Deo ${ }^{4}$, M. De Vincenzi ${ }^{39,40}$, A. Derbin ${ }^{31}$, A. Devoto ${ }^{9,8}$, F. Di Eusanio ${ }^{34}$, G. Di Pietro ${ }^{4,25}$, C. Dionisi ${ }^{35,37}$, E. Edkins ${ }^{15}$, A. Empl ${ }^{16}$, A. Fan ${ }^{47}$, G. Fiorillo ${ }^{29,28}$, K. Fomenko ${ }^{12}$, G. Forster ${ }^{48,1}$, D. Franco ${ }^{2}$, F. Gabriele ${ }^{4}$, C. Galbiati ${ }^{34,25}$, S. Giagu ${ }^{35,37}$, C. Giganti ${ }^{22}$, G. K. Giovanetti ${ }^{34}$, A. M. Goretti ${ }^{4}$, F. Granato ${ }^{44}$, L. Grandi ${ }^{10}$, M. Gromov ${ }^{27}$, M. Guan ${ }^{17}$, B. R. Hackett ${ }^{15}$, K. Herner ${ }^{1}$, D. Hughes ${ }^{34}$, P. Humble ${ }^{30}$, E. V. Hungerford ${ }^{16}$, Al. lanni ${ }^{23,4}$, An. lanni ${ }^{34,4}$, I. James ${ }^{39,40}$, T. N. Johnson ${ }^{45}$, C. Jollet ${ }^{43}$, K. Keeter ${ }^{11}$, C. L. Kendziora ${ }^{1}$, G. Koh ${ }^{34}$, D. Korablev ${ }^{12}$, G. Korga ${ }^{16,4}$, A. Kubankin ${ }^{6}$, X. Li ${ }^{34}$, M. Lissia ${ }^{8}$, B. Loer ${ }^{30}$, P. Lombardi ${ }^{25}$, G. Longo ${ }^{29,28}$, Y. Ma ${ }^{17}$, I. N. Machulin ${ }^{20,24}$, A. Mandarano ${ }^{3,4}$, S. M. Mari ${ }^{39,40}$, J. Maricic ${ }^{15}$, L. Marini ${ }^{14,13}$, C. J. Martoff ${ }^{44}$, A. Meregaglia ${ }^{43}$, P. D. Meyers ${ }^{34}$, R. Milincic ${ }^{15}$, J. D. Miller ${ }^{16}$, D. Montanari ${ }^{1}$, A. Monte ${ }^{48}$, B. J. Mount ${ }^{11}$, V. N. Muratova ${ }^{31}$, P. Musico ${ }^{13}$, J. Napolitano ${ }^{44}$, A. Navrer Agasson ${ }^{22}$, S. Odrowski ${ }^{4}$, M. Orsini ${ }^{4}$, F. Ortica ${ }^{33,32}$, L. Pagani ${ }^{14,13}$, M. Pallavicini ${ }^{14,13}$, E. Pantic ${ }^{45}$, S. Parmeggiano ${ }^{25}$, K. Pelczar ${ }^{19}$, N. Pelliccia ${ }^{33,32}$, A. Pocar ${ }^{48,34}$, S. Pordes ${ }^{1}$, D. A. Pugachev ${ }^{20,24}$, H. Qian ${ }^{34}$, K. Randle ${ }^{34}$, G. Ranucci ${ }^{25}$, M. Razeti ${ }^{8}$, A. Razeto ${ }^{4,34}$, B. Reinhold ${ }^{15}$, A. L. Renshaw ${ }^{16,47}$, M. Rescigno ${ }^{35}$, Q. Riffard ${ }^{2}$, A. Romani ${ }^{33,32}$, B. Rossi ${ }^{28,34}$, N. Rossi ${ }^{4}$, D. Rountree ${ }^{49}$, D. Sablone ${ }^{4}$, P. Saggese ${ }^{25}$, R. Saldanha ${ }^{10}$, W. Sands ${ }^{34}$, C. Savarese ${ }^{3,4}$, B. Schlitzer ${ }^{45}$, E. Segreto ${ }^{7}$, D. A. Semenov ${ }^{31}$, E. Shields ${ }^{34}$, P. N. Singh ${ }^{16}$, M. D. Skorokhvatov ${ }^{20,24}$, O. Smirnov ${ }^{12}$, A. Sotnikov ${ }^{12}$, C. Stanford ${ }^{34}$, Y. Suvorov ${ }^{47,4,20}$, R. Tartaglia ${ }^{4}$, J. Tatarowicz ${ }^{44}$, G. Testera ${ }^{13}$, A. Tonazzo ${ }^{2}$, P. Trinchese ${ }^{29,28}$, E. V. Unzhakov ${ }^{31}$, M. Verducci ${ }^{35,37}$, A. Vishneva ${ }^{12}$, B. Vogelaar ${ }^{49}$, M. Wada ${ }^{34}$, S. Walker ${ }^{29,28}$, H. Wang ${ }^{47}$, Y. Wang ${ }^{17,47}$, A. W. Watson ${ }^{44}$, S. Westerdale ${ }^{34}$, J. Wilhelmi ${ }^{44}$, M. M. Wojcik ${ }^{19}$, Xi. Xiang ${ }^{34}$, X. Xiao ${ }^{47}$, J. Xu ${ }^{34}$, C. Yang ${ }^{17}$, A. Zec ${ }^{48}$, W. Zhong ${ }^{17}$, C. Zhu ${ }^{34}$, G. Zuzel ${ }^{19}$ 
${ }^{1}$ Fermi National Accelerator Laboratory, Batavia, IL 60510, USA

${ }^{2}$ APC, Université Paris Diderot, CNRS/IN2P3, CEA/Irfu, Obs. de Paris, Sorbonne Paris Cité, Paris 75205, France

${ }^{3}$ Gran Sasso Science Institute, L'Aquila AQ 67100, Italy

${ }^{4}$ Laboratori Nazionali del Gran Sasso, Assergi AQ 67010, Italy

${ }^{5}$ Department of Physics, Augustana University, Sioux Falls, SD 57197, USA

${ }^{6}$ Radiation Physics Laboratory, Belgorod National Research University, Belgorod 308007, Russia

${ }^{7}$ Institute of Physics Gleb Wataghin, Universidade Estadual de Campinas, São Paulo 13083-859, Brazil

${ }^{8}$ Istituto Nazionale di Fisica Nucleare, Sezione di Cagliari, Cagliari 09042, Italy

${ }^{9}$ Department of Physics, Università degli Studi, Cagliari 09042, Italy

${ }^{10}$ Kavli Institute, Enrico Fermi Institute, and Dept. of Physics, University of Chicago, Chicago, IL 60637, USA

${ }^{11}$ School of Natural Sciences, Black Hills State University, Spearfish, SD 57799, USA

${ }^{12}$ Joint Institute for Nuclear Research, Dubna 141980, Russia

${ }^{13}$ Istituto Nazionale di Fisica Nucleare, Sezione di Genova, Genova 16146, Italy

${ }^{14}$ Department of Physics, Università degli Studi, Genova 16146, Italy

${ }^{15}$ Department of Physics and Astronomy, University of Hawai' $i$, Honolulu, HI 96822, USA

${ }^{16}$ Department of Physics, University of Houston, Houston, TX 77204, USA

${ }^{17}$ Institute of High Energy Physics, Beijing 100049, China

${ }^{18}$ Institute for Nuclear Research, National Academy of Sciences of Ukraine, Kiev 03680, Ukraine

${ }^{19}$ Smoluchowski Institute of Physics, Jagiellonian University, Krakow 30348, Poland

${ }^{20}$ National Research Centre Kurchatov Institute, Moscow 123182, Russia

${ }^{21}$ Lawrence Livermore National Laboratory, Livermore, CA 94550, USA

${ }^{22}$ LPNHE Paris, Université Pierre et Marie Curie, Université Paris Diderot, CNRS/IN2P3, Paris 75252, France

${ }^{23}$ Laboratorio Subterráneo de Canfranc, Canfranc Estación 22880, Spain

${ }^{24}$ National Research Nuclear University MEPhI, Moscow 115409, Russia

${ }^{25}$ Istituto Nazionale di Fisica Nucleare, Sezione di Milano, Milano 20133, Italy

${ }^{26}$ Department of Physics, Università degli Studi, Milano 20133, Italy

${ }^{27}$ Skobeltsyn Institute of Nuclear Physics, Lomonosov Moscow State University, Moscow 119991, Russia

${ }^{28}$ Istituto Nazionale di Fisica Nucleare, Sezione di Napoli, Napoli 80126, Italy

${ }^{29}$ Department of Physics, Università degli Studi Federico II, Napoli 80126, Italy

${ }^{30}$ Pacific Northwest National Laboratory, Richland, WA 99354, USA

${ }^{31}$ St. Petersburg Nuclear Physics Institute NRC Kurchatov Institute, Gatchina 188350, Russia

${ }^{32}$ Istituto Nazionale di Fisica Nucleare, Sezione di Perugia, Perugia 06123, Italy

${ }^{33}$ Department of Chemistry, Biology and Biotechnology, Università degli Studi, Perugia 06123, Italy

${ }^{34}$ Department of Physics, Princeton University, Princeton, NJ 08544, USA

${ }^{35}$ Istituto Nazionale di Fisica Nucleare, Sezione di Roma Uno, Roma 00185, Italy

${ }^{37}$ Department of Physics, Università degli Studi "La Sapienza"di Roma, Roma 00185, Italy

${ }^{38}$ Physics Department, Università degli Studi “La Sapienza”di Roma, Roma 00185, Italy

${ }^{39}$ Istituto Nazionale di Fisica Nucleare, Sezione di Roma Tre, Roma 00146, Italy

${ }^{40}$ Department of Physics and Mathematics, Università degli Studi Roma Tre, Roma 00146, Italy

${ }^{41}$ Instituto de Física, Universidade de São Paulo, São Paulo 05508-090, Brazil

${ }^{42}$ SLAC National Accelerator Laboratory, Menlo Park, CA 94025, USA

${ }^{43}$ IPHC, Université de Strasbourg, CNRS/IN2P3, Strasbourg 67037, France

${ }^{44}$ Department of Physics, Temple University, Philadelphia, PA 19122, USA

${ }^{45}$ Department of Physics, University of California, Davis, CA 95616, USA

${ }^{46}$ School of Physics, University of Chinese Academy of Sciences, Beijing 100049, China

${ }^{47}$ Department of Physics and Astronomy, University of California, Los Angeles, CA 90095, USA

${ }^{48}$ Amherst Center for Fundamental Interactions and Dept. of Physics, University of

Massachusetts, Amherst, MA 01003, USA

${ }^{49}$ Department of Physics, Virginia Tech, Blacksburg, VA 24061, USA

E-mail: yguardindfnal.gov 
DarkSide-50 is a direct dark matter search experiment operating underground at Laboratori Nazionali del Gran Sasso. It is based on a Time Projection Chamber (TPC) that contains $50 \mathrm{~kg}$ of liquid argon; the TPC cryostat sits inside an active neutron veto based on a boron-loaded organic scintillator, which is in turn installed inside a water Cherenkov muon veto. The experiment started acquiring data in Nov 2013 filled with atmospheric argon. In April 2015 it commissioned the low-radioactivity argon from underground sources and has been running in a stable manner since then. We report the current status of the detector and the latest results, including the measurement of the radioactivity of the underground argon and the most sensitive dark matter search performed with an argon target.

38th International Conference on High Energy Physics

3-10 August 2016

Chicago, USA

* Speaker. 


\section{Introduction}

The DarkSide program goal is to detect signals of dark matter (DM) particles, by using liquid argon (LAr) as a target in a dual-phase Time Projection Chamber (TPC) with active background suppression systems. In order to achieve the goal, various technologies are adopted to reduce and reject background events.

The main motivation to use LAr is its strong pulse shape discrimination (PSD) of electron recoils (ERs) from nuclear recoils (NRs). Considering that the dominant mechanism to detect an interaction between heavy DM $\left(>10 \mathrm{GeV} / \mathrm{c}^{2}\right)$ and baryonic matter is expected to be elastic scattering off nuclei, PSD in LAr provides a tool to reject the dominant background of ERs. The results from DarkSide-50 with atmospheric argon (AAr) data show that using PSD, a total of $1.5 \times 10^{7}$ ER events can be completely rejected in the DM energy region of interest (ROI), leaving no events after an exposure of $(1422 \pm 67) \mathrm{kg}$ day [1]. The use of a dual-phase TPC allows 3D position reconstruction which can be used to remove surface background events by fiducializing the detection volume. Furthermore, the ratio of ionization yield to scintillation yield can provide additional discrimination power against ER backgrounds. A challenge of employing LAr as a target for DM detection comes from the presence of the $\beta$-emitting isotope ${ }^{39} \mathrm{Ar}$ in AAr at the level of $1 \mathrm{~Bq} / \mathrm{kg}$. Since ${ }^{39} \mathrm{Ar}$ has a decay half-life of 269 years and is mainly generated by cosmic rays in the atmosphere, underground argon sources can be free of ${ }^{39} \mathrm{Ar}$ or have only trace amounts activated by radiation from the rock.

\section{DarkSide-50 Detectors}

DarkSide-50 consists of three nested detectors: the dual-phase argon TPC, with a LAr target for DM detection; the organic Liquid Scintillator Veto (LSV) provides rejection of cosmogenic and radiogenic neutrons, and $\gamma \mathrm{s}$ from the detector materials and the Water Cherenkov Detector (WCD), which rejects cosmic muons and suppresses radiogenic background from surrounding rocks and detectors. An incoming DM particle which scatters from an Ar nucleus would result in excitation and ionization of the argon. The excited Ar dimers de-excite and emit a first scintillation signal, S1, at the vertex of the recoil. The ionization electrons which escape recombination are drifted toward the top of the TPC by an electric field of $200 \mathrm{~V} / \mathrm{cm}$ and are extracted into a gas phase layer, where a higher electric field generates the second scintillation light, S2, by gas proportional scintillation. All the inner surfaces of the TPC are coated with TPB to convert the $128 \mathrm{~nm}$ argon scintillation light into the visible range. The scintillation photons are detected with two arrays of 19 PMTs on the top and bottom of the TPC. The time difference between S1 and S2 gives the depth (z position) of the event, and the transverse $(\mathrm{x}, \mathrm{y})$ position is reconstructed from the distribution pattern of S2 light among the top PMTs. The LSV has an array of 110 8" PMTs attached on the inside of a $4 \mathrm{~m}$ diameter stainless steel sphere filled with liquid scintillator. The scintillator is a mixture of pseudocumene (PC) and trimethyl borate (TMB) with a wavelength shifter, diphenyloxazole (PPO). Neutrons are detected in the LSV via elastic scattering signals and/or capture signals, mainly on ${ }^{10} \mathrm{~B}$ in the TMB. The veto efficiency of NRs from capture signals has been studied and estimated to be $>99.1 \%$ using an AmBe neutron source and MC simulation [2]. The WCD has an array of 80 8" PMTs mounted on the inside of an $11 \mathrm{~m}$ diameter by $10 \mathrm{~m}$ high cylindrical tank filled with high purity water. 


\section{Dark Matter Search with an Underground Ar Target}

The underground argon (UAr) was extracted from a stream of gas from a $\mathrm{CO}_{2}$ well in Cortez, Colorado, which also contained other gases including $\mathrm{N}_{2}$ and $\mathrm{He}$. Most of the $\mathrm{CO}_{2}$ and $\mathrm{N}_{2}$ was removed on site and a mixture of $\sim 5 \% \mathrm{Ar}, \sim 5 \% \mathrm{~N}_{2}$, and $90 \%$ He was sent to Fermilab for purification by cryogenic distillation [3]. DarkSide-50 TPC was filled with the purified UAr at LNGS in Italy on April 1, 2015. To determine the ${ }^{39} \mathrm{Ar}$ depletion factor, the UAr data was compared to a GEANT4-based MC simulation. The MC was tuned on high statistic AAr data and validated against several $\gamma$ calibration sources. The ${ }^{39} \mathrm{Ar}$ and ${ }^{85} \mathrm{Kr}$ activities were measured from a simultaneous fit of the MC to S1 data taken with the field off (See Fig. 1) and field-on $(200 \mathrm{~V} / \mathrm{cm}$ ) data. The ${ }^{39} \mathrm{Ar}$ and ${ }^{85} \mathrm{Kr}$ activities in the UAr were determined to be $(0.73 \pm 0.11) \mathrm{mBq} / \mathrm{kg}$ and $(2.05 \pm 0.13) \mathrm{mBq} / \mathrm{kg}$ respectively. The ${ }^{39} \mathrm{Ar}$ activity of the UAr corresponds to a reduction by a factor of $(1.4 \pm 0.2) \times 10^{3}$ relative to AAr.

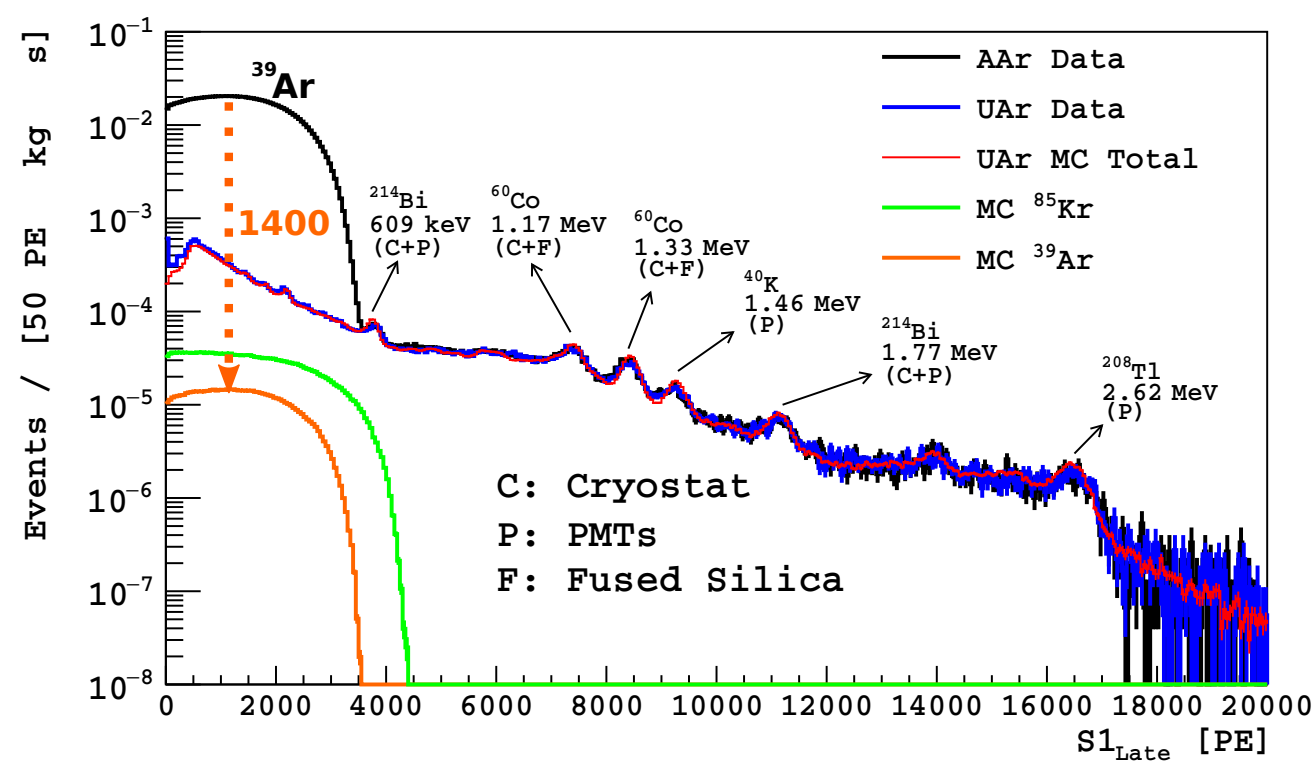

Figure 1: Comparison of the measured field off spectra for the UAr and AAr targets, normalized to exposure. Also shown are the MC fit to the UAr data and individual components of ${ }^{85} \mathrm{Kr}$ and ${ }^{39} \mathrm{Ar}$ extracted from the fit. Several $\gamma$ lines are identified from ${ }^{238} \mathrm{U},{ }^{232} \mathrm{Th},{ }^{40} \mathrm{~K}$, and ${ }^{60} \mathrm{Co}$ present in the detector construction materials.

The presence of ${ }^{85} \mathrm{Kr}$ in UAr was unexpected. To confirm its presence an independent estimate of the ${ }^{85} \mathrm{Kr}$ decay rate in UAr was obtained from the data, by identifying $\beta-\gamma$ coincidences from the $0.43 \%$ decay branch to ${ }^{85 m} \mathrm{Rb}$ with mean lifetime $1.464 \mu$ s. In Fig. 2 the distribution of times between ${ }^{85} \mathrm{Kr}^{85 m} \mathrm{Rb}$ events is shown. The ${ }^{85} \mathrm{Kr}$ activity was found to be $(1.92 \pm 0.05) \mathrm{mBq} / \mathrm{kg}$, which is consistent with the measurement obtained from the energy spectrum fit. There has been no attempt to remove krypton from the UAr, although cryogenic distillation would likely perform very effectively.

The DM search result with UAr [5] is based on the data set acquired from April 8 to July 31 in 2015 (70.9 live-days after data quality cuts) at a drift field of $200 \mathrm{~V} / \mathrm{cm}$. The light yield of S1 


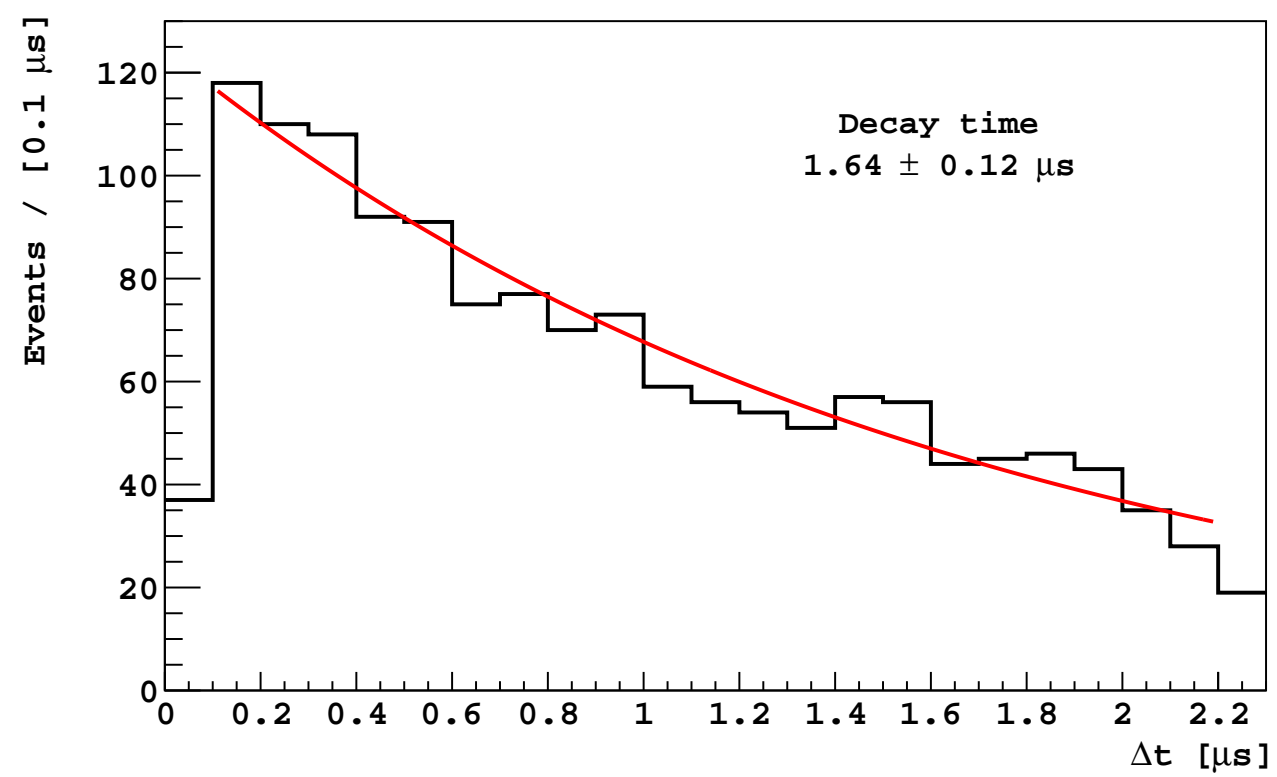

Figure 2: Distribution of time intervals between $\beta-\gamma$ events for ${ }^{85} \mathrm{Kr}^{85 m} \mathrm{Rb}$ candidate events. Candidate events are extracted from one single LAr TPC event, and the fit window is restricted to the interval in which the extraction algorithm is fully efficient, i.e. $0.1 \mu \mathrm{s}$ to $2.2 \mu \mathrm{s}$. The delayed coincidence time of $(1.64 \pm 0.12)$ $\mu$ s should be compared with the ${ }^{85 m} \mathrm{Rb}$ lifetime of $1.464 \mu \mathrm{s}$ [4].

signals was determined to be $(8.1 \pm 0.2) \mathrm{PE} / \mathrm{keV}$ at zero field from the ${ }^{83 m} \mathrm{Kr}$ peak, consistent with the light yield in AAr. A set of criteria used to select candidate DM recoils, i.e. single NRs in the fiducial volume, are described in detail in Ref. [5]. The PSD parameter used in this analysis is f90, the fraction of S1 light detected in the first $90 \mathrm{~ns}$ of the pulse. The DM ROI is defined in the $900 \mathrm{vs}$ S1 plane with a $90 \%$ NR acceptance contour derived from the 990 response in SCENE [6], and a leakage curve corresponding to a total predicted leakage of $<0.1$ events during the exposure. The final event distribution in the $\mathrm{f} 90$ vs S1 plane is presented in Fig. 3, and there are no events in the DM ROI. Given the background-free null result, a 90\% C.L. exclusion curve in the WIMP mass vs. WIMP nucleon cross section plane was derived, which is shown in Fig. 4.

\section{Conclusion}

The DarkSide-50 detector achieved the most sensitive limit on DM-nucleon cross section among experiments using LAr. The ${ }^{39}$ Ar depletion factor in UAr was determined to be $(1.4 \pm 0.2) \times 10^{3}$ relative to AAr. The combination of the null result of the UAr [5] and AAr [1] exposures set an upper limit on the WIMP-nucleon spin-independent cross section of $2.0 \times 10^{44} \mathrm{~cm}^{2}$ on the DMnucleon cross section at $100 \mathrm{GeV} / \mathrm{c}^{2} \mathrm{DM}$ mass.

The DarkSide collaboration is moving towards a next-generation detector. Given the exceptional PSD power of LAr and high depletion factor of UAr, a direct DM search using a LAr TPC with a fiducial mass of 20 tonne of depleted argon has been proposed. In order to suppress both NR 


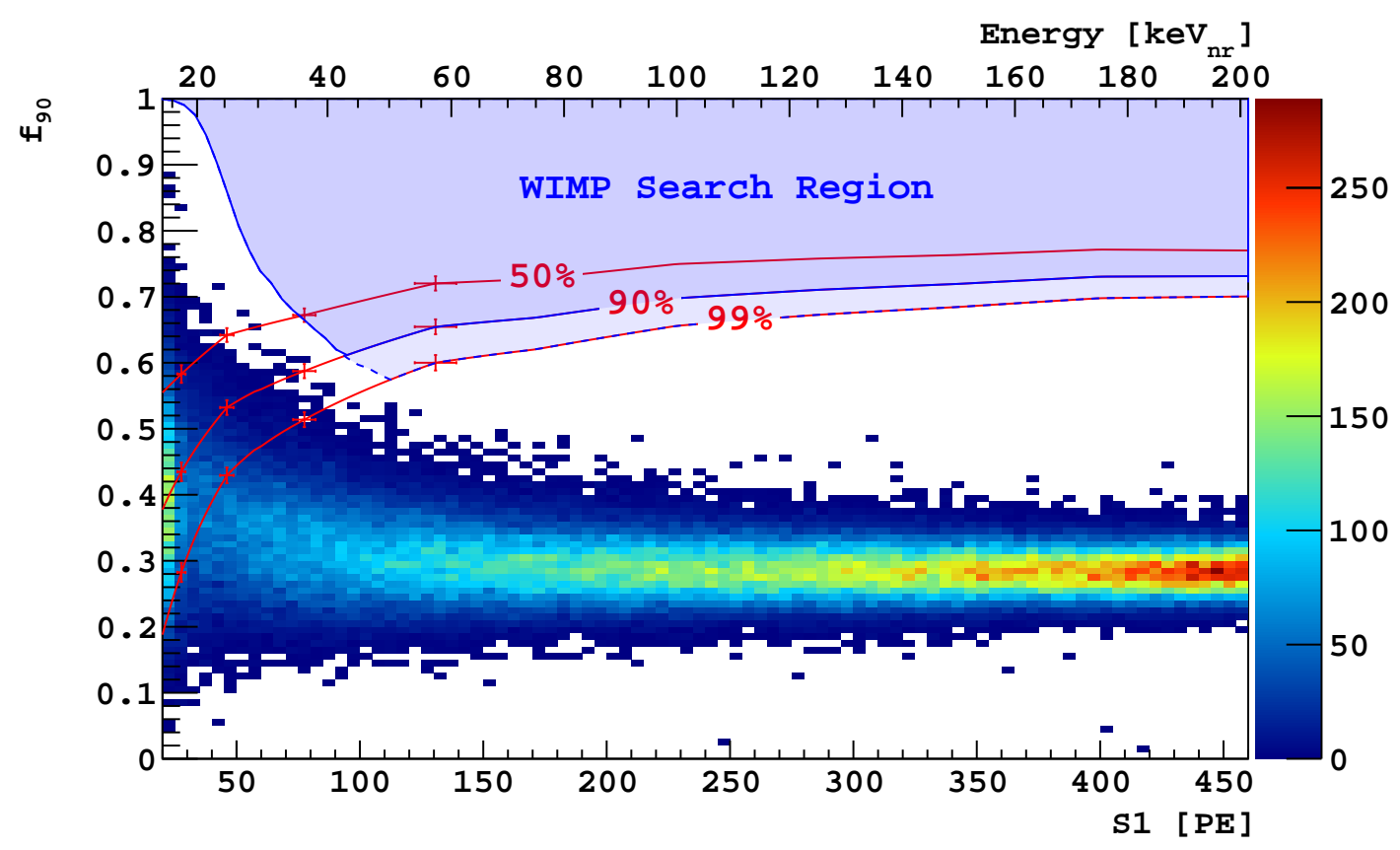

Figure 3: Distribution of events in the $\mathrm{f} 90$ vs $\mathrm{S} 1$ plane after applying all cuts in the energy region of interest. Shaded blue with solid blue outline: DM search region. The f90 acceptance contours are drawn by connecting the red points derived from the SCENE measurements [6] and extending the contours using DarkSide-50 AmBe data.

and ER backgrounds from conventional PMTs and improve light yield, silicon photomultipliers are planned to be used and are currently under development. To acquire the required amount of low-radioactivity argon with a sufficient depletion factor, two key projects are planned: Urania is an enlarged argon extraction plant in Cortez, Colorado with expected capacity of $100 \mathrm{~kg} / \mathrm{day}$ of UAr; and Aria consists of two $350 \mathrm{~m}$ tall distillation columns to be sited in a shaft at the Serucci mine in Sardinia. Aria is capable of reducing ${ }^{39} \mathrm{Ar}$ in UAr further with additional depletion factor between 10 to 100 .

\section{References}

[1] Agnes, P. et al. [DarkSide Collaboration], "First results from the DarkSide-50 dark matter experiment at Laboratori Nazionali del Gran Sasso", Phys. Lett. B 743, 456 (2015).

[2] Agnes, P. et al. [DarkSide Collaboration], "The veto system of the DarkSide-50 experiment”, J. Inst. 11, P03016 (2016).

[3] Back, H. O. et al., "First Large Scale Production of Low Radioactivity Argon From Underground Sources", arXiv:1204.6024 (2012).

[4] R. B. Firestone, C. M. Baglin, and S. Y. F. Chu, Table of isotopes, Wiley-Interscience (1999).

[5] Agnes, P. et al. [DarkSide Collaboration], "Results from the first use of low radioactivity argon in a dark matter search”, Phys. Rev. D 93, 081101 (2016). 


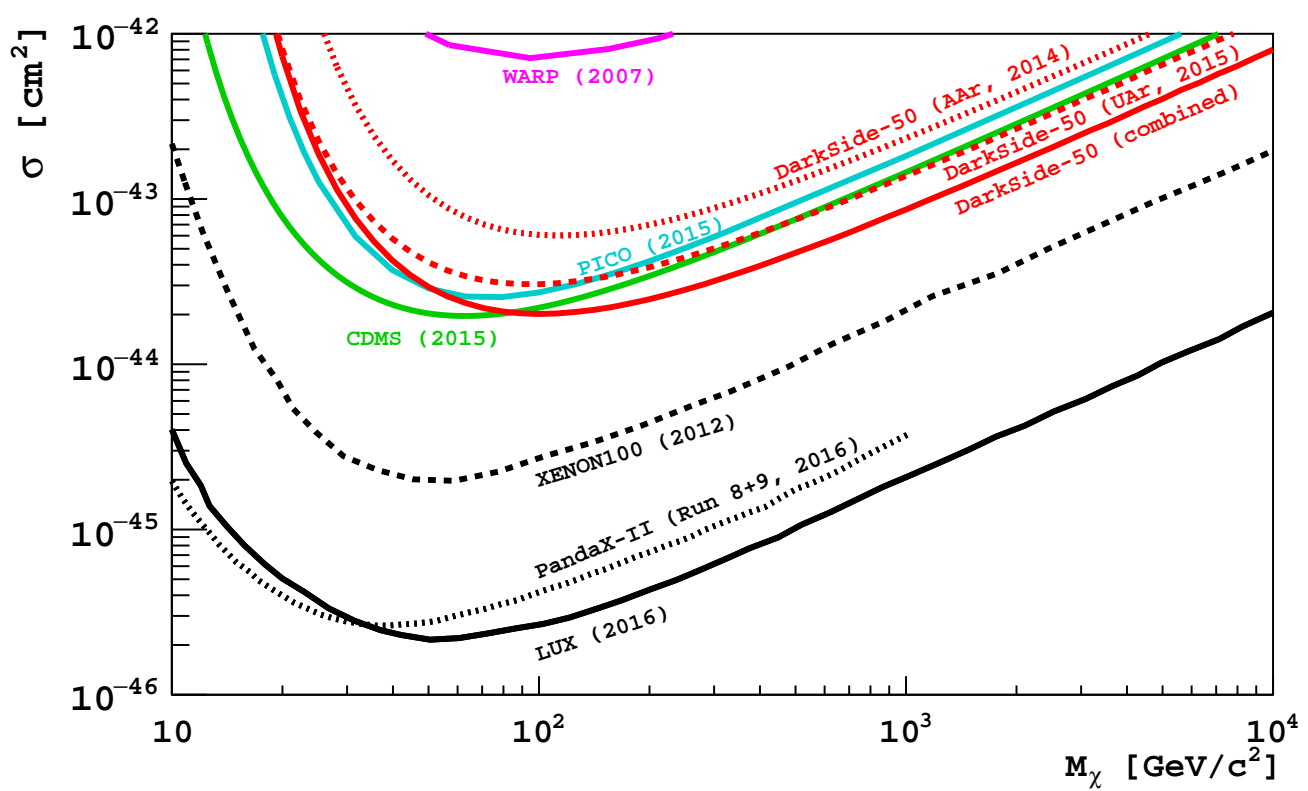

Figure 4: Spin-independent DM-nucleon cross section 90\% C.L. exclusion plots for the DarkSide-50 AAr [1], UAr [5] and combination of both campaigns. Also shown are results from other experiments for comparison $[7,8,9,10,11,12]$.

[6] Cao, H. et al. [SCENE Collaboration], "Measurement of scintillation and ionization yield and scintillation pulse shape from nuclear recoils in liquid argon”, Phys. Rev. D 91, 092007 (2015).

[7] Benetti, P. et al. [WArP Collaboration], "First results from a dark matter search with liquid argon at 87 $\mathrm{K}$ in the Gran Sasso underground laboratory", Astropart. Phys. 28, 495 (2008).

[8] Amole C. et al. [PICO Collaboration], "Dark Matter Search Results from the PICO-2L $\mathrm{C}_{3} \mathrm{~F}_{8}$ Bubble Chamber”, Phys. Rev. Lett. 114, 231302 (2015).

[9] Agnese R. et al. [SuperCDMS Collaboration], "Improved WIMP-search reach of the CDMS II germanium data”, Phys. Rev. D 92, 072003 (2015).

[10] Aprile E. et al. [XENON100 Collaboration], "Dark Matter Results from 225 Live Days of XENON100 Data”, Phys. Rev. Lett. 109, 181301 (2012).

[11] Tan A. et al. [PandaX Collaboration], "Dark Matter Results from First 98.7-day Data of PandaX-II Experiment”, Phys. Rev. Lett. 117, 121303 (2016)

[12] Akerib D. S. et al. [LUX Collaboration], "Results from a search for dark matter in the complete LUX exposure", arXiv:1608.07648 (2016). 